\title{
Central Angiotensin I Increases Swallowing Activity and Oxytocin Release in the Near-Term Ovine Fetus
}

\author{
Lijun Shi $^{\mathrm{a}}$ Caiping Mao ${ }^{\mathrm{a}}$ Fanxing Zeng ${ }^{\mathrm{a}}$ Lubo Zhang ${ }^{\mathrm{a}}$ b Zhice $\mathrm{Xu}^{\mathrm{a}} \mathrm{b}$ \\ a Beijing Sport University, Beijing, The First Hospital of Soochow University, Suzhou, PR China; \\ ${ }^{\mathrm{b}}$ Center for Perinatal Biology, Loma Linda University School of Medicine, Loma Linda, Calif., USA
}

\section{Key Words}

Angiotensin I· Swallowing $\cdot$ Oxytocin $\cdot$ Fetus

\begin{abstract}
The brain renin-angiotensin system (RAS) plays an important role in hydromineral and neuroendocrine balance. Although previous studies showed that exogenous angiotensin (Ang) II increased dipsogenic and vasopressin responses in nearterm fetuses, little is known about the functional development of fetal endogenous brain RAS in the regulation of body fluid homeostasis. To determine the functional development of the central angiotensin-converting enzyme (ACE) in utero, we investigated the electrocortical (ECoG) activity, swallowing activity, oxytocin (OT) release, and c-fos expression in response to intracerebroventricular Ang I administration in the near-term fetal lamb. Ang I did not change fetal low-voltage (LV) and high-voltage (HV) ECoG temporal distributions, but increased fetal swallowing activity during LV ECoG (1.0 \pm 0.1 to $3.5 \pm 0.4$ swallows/min). Additionally, Ang I evoked an increase in c-fos-immunoreactivity in putative dipsogenic centers, including the supraoptic and paraventricular nuclei of the hypothalamus, accompanied by an increase in fetal plasma OT levels. The expression of c-fos was demonstrated in OT neurons in the hypothalamus. The Ang I-mediated increase in fetal swallowing and plasma OT was
\end{abstract}

inhibited by captopril. These results demonstrate the functional development of the fetal brain ACE system in the last trimester of gestation, which plays an important role in the RAS-mediated dipsogenic response and OT release in the regulation of body fluid homeostasis.

Copyright $\odot 2011$ S. Karger AG, Basel

\section{Introduction}

The brain renin-angiotensin system (RAS) plays an important role in the regulation of hydromineral balance [1]. In adult animals, a central administration of angiotensin (Ang) II has been shown to produce thirst, a reduction in the salt appetite, and the release of the neurohypophysial hormones vasopressin (VP) and oxytocin (OT) [2-4]. However, the development of a functional RAS system in the fetal brain, especially the brain endogenous RAS in utero remains unclear. For example, the physiological function of the angiotensin-converting enzyme (ACE) in the fetal brain is unknown, but it is known to exist in the fetal brain [5-7]. In the ovine fetus, stimulated swallowing has been demonstrated in response to dipsogenic challenges [8]. However, fetuses swallow the amniotic fluids in utero that contain water, electrolytes, and other materials. It is technically difficult to distin-

\section{KARGER}

Fax +41613061234

E-Mail karger@karger.ch

www.karger.com
(C) 2011 S. Karger AG, Basel

0028-3835/12/0953-0248\$38.00/0

Accessible online at:

www.karger.com/nen
Zhice Xu

Institute for Fetal-Origin Diseases, The First Hospital of Soochow University 188 Shizhi Rd.

Suzhou 215007 (PR China)

Tel. +86 5126185 9998, E-Mail zxu@llu.edu 
guish salt intake from water intake in the fetus. OT is an important hormone in the regulation of salt appetite [9, 10], and previous studies demonstrated that the central Ang II treatment not only stimulated OT release but also activated central OT pathways that were inhibitory to salt appetite, thus limiting the consumption of saline [11]. Our recent work has demonstrated that central Ang II increases VP release and the neural activity in the VP neurons in the fetal hypothalamus $[12,13]$, indicating that the central RAS-mediated fetal neuropeptide network is functional. Unlike VP, which acts to regulate vascular and renal function, OT has unique central functions to regulate salt intake beside its peripheral functions. Because it is difficult to study fetal salt appetite by measuring swallowing behavior alone, we have modified the experimental strategy and focused on the control center in the brain and measurement of OT release, and the function of OT cells in addition to detecting fetal behavioral responses.

Accordingly, the present study was designed to determine the functional responses of fetal brain ACE in relation to swallowing and OT release in utero. We hypothesized that the endogenous ACE in the fetal brain is functionally developed by the third trimester of gestation, and it is able to convert exogenous Ang I into Ang II and to induce Ang-II-like dipsogenic responses and Ang-IIstimulated OT release in the near-term fetal lamb.

\section{Animals and Methods}

\section{Animals and Surgical Preparation}

Fifteen time-dated pregnant ewes (gestational age $125 \pm 5$ days, term $\sim 145$ days, singleton) were used. Animals were housed indoors in individual cages and acclimated to a 12:12-hour lightdark cycle. Both food and water were provided ad libitum. All protocols in this study were approved by the institutional animal care committee. Anesthesia was induced by an intramuscular injection of ketamine hydrochloride $(20 \mathrm{mg} / \mathrm{kg})$ and atropine sulfate $(50$ $\mu \mathrm{g} / \mathrm{kg}$ ) and was maintained by maternal endotracheal ventilation with $1 \mathrm{l} / \mathrm{min}$ oxygen and $3 \%$ isoflurane. Polyethylene catheters (ID $=1.8 \mathrm{~mm}, \mathrm{OD}=2.3 \mathrm{~mm}$ ) were inserted into the maternal femoral vein and artery and advanced into the inferior vena cava and abdominal aorta, respectively. The uterus was exposed by a midline abdominal incision, and a small hysterotomy was performed to provide access to fetal hind limbs. Polyethylene catheters $(\mathrm{ID}=1.0 \mathrm{~mm}, \mathrm{OD}=1.8 \mathrm{~mm}$ ) were inserted into the fetal femoral vein and artery. Bipolar electromyography (EMG) electrodes were placed on the fetal thyrohyoid muscle and upper and lower esophagus to determine the swallowing activity, as previously reported $[14,15]$. Electrodes were also implanted on the parietal dura through two burr holes for the determination of the fetal electrocortical (ECoG) activity. An intracranial cannula (18 gauge) was placed in the lateral ventricle and immobilized with dental cement with the assistance of two stainless steel screws affixed in the fetal skull. The coordinates for the cannula placement were: anteriorposterior: $+0.1 \mathrm{~cm}$ in the front of bregma; medial-lateral: $0.8 \mathrm{~cm}$ from the middle line, and ventral: $1.8 \mathrm{~cm}$ below the dura. Patency of the catheter at insertion was assessed by free flow of cerebrospinal fluid via gravity drainage. The uterine incisions were closed in layers. All catheters were passed through a subcutaneous tunnel and exteriorized through a small incision on the ewe's flank and placed in a cloth pouch. Four to 5 days of postoperative recovery were allowed before the experiments commenced. Antibiotics were administered by daily intravenous injection to the ewe $(70 \mathrm{mg}$ gentamicin and $1 \mathrm{~g}$ oxacillin) and to the fetus (5 $\mathrm{mg}$ gentamicin and $30 \mathrm{mg}$ oxacillin) for 3-4 days postsurgically.

\section{Behavioral and Physiological Experiments}

All experiments were performed on conscious animals standing in their holding cages, with food and water provided ad libitum. Studies began with a basal period ( -60 to $0 \mathrm{~min}$ ) followed by an experimental period ( 0 to $120 \mathrm{~min}$ ) in three groups $(\mathrm{n}=5 /$ group). In group 1 , beginning at time $0,1 \mathrm{ml}$ of isotonic saline (vehicle) was intracerebroventricularly (i.c.v.) injected into the fetus over $5 \mathrm{~min}$. In group 2, Ang I ( $5 \mu \mathrm{g} / \mathrm{kg}$; Sigma) in $1 \mathrm{ml}$ of isotonic saline was injected. In group 3, at $-10 \mathrm{~min}$, a bolus of captopril $(1.25 \mathrm{mg} / \mathrm{kg})$ was i.c.v. injected, and at $0 \mathrm{~min}$, another bolus of captopril $(1.25 \mathrm{mg} / \mathrm{kg})$ with Ang I $(5 \mu \mathrm{g} / \mathrm{kg})$ was injected. The doses of Ang I and captopril were chosen based on the previous reports [16] and our preliminary studies. Throughout the study, fetal swallowing and ECoG were measured continuously using a PowerLab Physiological Record with Chart 5 software (AD Instruments, Australia).

\section{Endocrine Experiments}

Throughout the basal and experimental periods, maternal and fetal arterial blood was withdrawn at timed intervals for the measurement of $\mathrm{pH}$, blood gases, hematocrit, plasma electrolyte composition, osmolality, and OT concentrations. Fetal blood samples were replaced with an equivalent volume of heparinized maternal blood withdrawn before the study. Blood $\mathrm{PO}_{2}, \mathrm{PCO}_{2}$, and $\mathrm{pH}$ were measured with a Nova analyzer (Model pHOx Plus L; Nova Biochemical, Waltham, Mass., USA) adjusted to the internal temperature of the sheep $\left(39^{\circ} \mathrm{C}\right)$. Plasma osmolality was measured by freezing-point depression on an Advanced Digimatic Osmometer. Plasma OT levels were analyzed by radioimmunoassay following Sep-Pak C18 cartridge (Waters Associates, Milford, Mass., USA) extraction. Samples for OT extraction were acidified with $1 \mathrm{~N} \mathrm{HCl}$ and were added slowly to the columns, which were then washed with $0.1 \%$ trifluoroacetic acid. The absorbed OT was eluted with $50 \%$ methanol and $0.1 \%$ TFA, and the eluates were dried in a Speed-Vac concentrator. The assay sensitivity was $1.6 \mathrm{pg} / \mathrm{tube}$ for and the intra- and inter-assay coefficients of variations were 8 and $12 \%$, respectively. OT recoveries averaged $70 \%$. All plasma samples were processed together.

\section{Immunostaining Experiments}

At the conclusion of the experiment, ewes were anesthetized under ketamine anesthesia $(20 \mathrm{mg} / \mathrm{kg}$ i.m.) and ventilated with a mixture of isofluorane and oxygen (both the mother and fetus were under the condition of anaesthesia). A midline abdominal incision was made, and the fetal head and neck were exposed. A 16-gauge needle was inserted into one fetal carotid artery for perfusion. The 
fetuses were perfused via the carotid artery with $0.01 \mathrm{M}$ phosphatebuffered saline followed by $4 \%$ paraformaldehyde (PFA) in $0.1 \mathrm{M}$ phosphate buffer. Immediately after perfusion, the fetal brain was removed and collected for histological studies. Postfixation was performed in paraformaldehyde solution for $12-24 \mathrm{~h}$, after which the brains were placed in $20 \%$ sucrose in $0.01 \mathrm{M}$ phosphate overnight. Coronal sections $(20 \mu \mathrm{m})$ of fetal brains were cut on a cryostat and every second section of the median preoptic nucleus (MnPO), organum vasculosum of the lamina terminalis (OVLT), subfornical organ (SFO), supraoptic nucleus (SON), and paraventricular nucleus (PVN) was used for c-fos immunoreactivity (Fosir) staining using the avidin-biotin-peroxidase technique. The tissue sections were incubated on a gentle shaker overnight at $4^{\circ} \mathrm{C}$ in the Fos primary antibody (1:10,000; Santa Cruz Biotech, Santa Cruz, Calif., USA) raised in rabbit. The sections were then incubated in a goat anti-rabbit serum $(1: 1,000)$ for $1 \mathrm{~h}$ and then processed using the Vectastain ABC kit (Vector Laboratories, Burlingame, Calif., USA) for $1 \mathrm{~h}$ at room temperature. The sections were then treated with $1 \mathrm{mg} / \mathrm{ml}$ diaminobenzidine tetrahydrochloride (0.02\% hydrogen peroxide). All sections were mounted on slides, dehydrated in alcohol, and then coverslipped.

Fos-ir and OT-immunoreactivity (OT-ir) double labelling (Fos-ir labelling in the nucleus and OT-ir labelling in the cytoplasm). Fos-ir and OT-ir double labeling was performed on tissue sections from the SON and PVN. Vectastain ABC kit was used for immunostaining with OT antibody (1:5,000; Diasorin, Stillwater, Minn., USA). The sections were incubated with the OT primary antibody overnight, and then anti-rabbit antibody- (1:200; Vector Laboratories) conjugated fluorescein-isothiocyanate was applied. All stained sections were mounted on slides, dehydrated in alcohol, and then coverslipped. The sections were observed with a darkfield microscope with a filter for fluorescein-isothiocyanate excitation for incident light fluorescence.

\section{Data Analysis}

All signals were analyzed by computer analysis of waveforms using a PowerLab system with Chart 5 software (AD Instruments, Australia). An EMG-propagated swallow, representing a coordinated laryngeal-esophageal contraction, was defined by a time sequence of integrated EMG signals from the thyrohyoid muscle to the upper and lower nuchal esophagus [17]. Fetal ECoG activity was assessed by visual analysis and was divided into periods of low voltage (LV) and high voltage (HV). Periods of ECoG activity that did not belong to either LV or HV activity were considered to be intermediate ECoG ( $<5 \%$ of total ECoG). Intermediate ECoG constituted less than $5 \%$ of the total ECoG activity and data from these periods were not analyzed. Total swallowing activity was calculated as defined above and expressed as swallows $/ \mathrm{min}$. The percentage of swallows associated with each ECoG state was then calculated. The number of Fos-ir positive cells in the brain was evaluated in a quantitative and blinded manner under Nikon fluorescent microscopy. Every other section was observed and the number of labelled cells was counted. SPSS software was used for statistical analysis.

All data are expressed as means \pm SEM. A repeated measures ANOVA was used to determine differences over time and effects of the treatments. Comparison before and after the treatments was determined with one-way ANOVA followed by the Tukey post hoc test. A p value of $<0.05$ or 0.01 was the probability level used to define statistical significance.

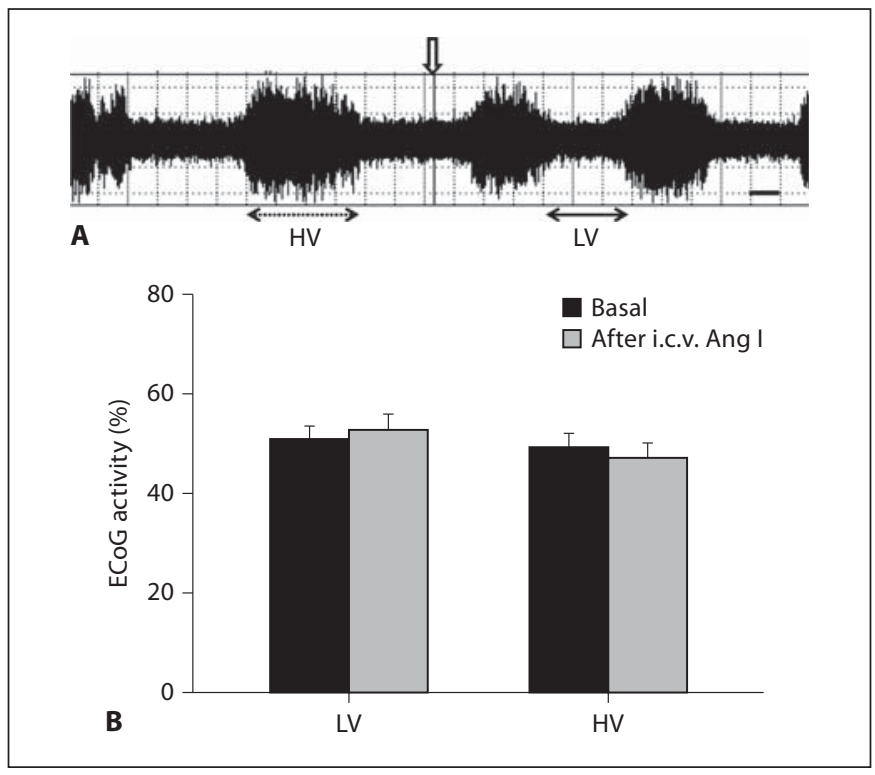

Fig. 1. A 60-min tracing depicting fetal LV and HV ECoG (A). Percentage time of fetal ECoG before and after i.c.v. Ang I (B). Scale bar $=5 \mathrm{~min}$. Ang I, $5 \mu \mathrm{g} / \mathrm{kg}$. The dotted arrow shows $\mathrm{HV}$ period, and the solid arrow shows LV period. The open arrow indicates the time for i.c.v. injection of Ang I.

\section{Results}

\section{Blood Values}

Histological analysis confirmed that all cannulae were inserted into the fetal lateral ventricle. I.c.v. injection of vehicle, Ang I, or captopril with Ang I had no effect on plasma osmolality, $\mathrm{Na}+, \mathrm{K}+, \mathrm{Cl}$ - concentrations, arterial blood $\mathrm{pH}, \mathrm{PO}_{2}, \mathrm{PCO}_{2}$, hemoglobin, and hematocrit in either the mother or the fetus (all $\mathrm{p}>0.05$ ). All arterial values were within normal ranges and were similar in control (i.c.v. injection of vehicle) and experimental groups (i.c.v. injection of Ang I or captopril + Ang I).

\section{ECoG Activity and Swallowing Activity}

There was no difference in the temporal distribution of the LV and HV states during the basal period in the animals of all the three groups, i.e. i.c.v. vehicle, Ang I, and captopril with Ang I. No difference in percentage of time spent in LV and HV ECoG was observed before and after i.c.v. injection of vehicle, Ang I (fig. 1), or captopril with Ang I. During the basal period in the control and experimental fetal groups, normal swallowing rates were observed during LV and HV ECoG. The majority of fetal swallowing activity occurred during LV ECoG periods. In the control animals, fetal swallowing did not 
Fig. 2. A 60-second tracing depicting fetal swallowing activity after i.c.v. vehicle (A), Ang I (B) or captopril with Ang I (C) during LV ECoG. The first, second and third panel in A, B and $\mathbf{C}$ represent the ECoG of thyrohyoid muscle, nuchal esophagus, and thoracic esophagus, respectively. D Fetal swallowing activity during LV ECoG (a) and HV ECoG (b) before and after i.c.v. injection of vehicle, Ang I or captopril with Ang I. 0 min represents the time of i.c.v. injection. ${ }^{*} \mathrm{p}<0.01$ compared with the basal level. Ang I, $5 \mu \mathrm{g} / \mathrm{kg}$; captopril, 2.5 $\mathrm{mg} / \mathrm{kg}$. Scale bar $=8 \mathrm{~s}$.

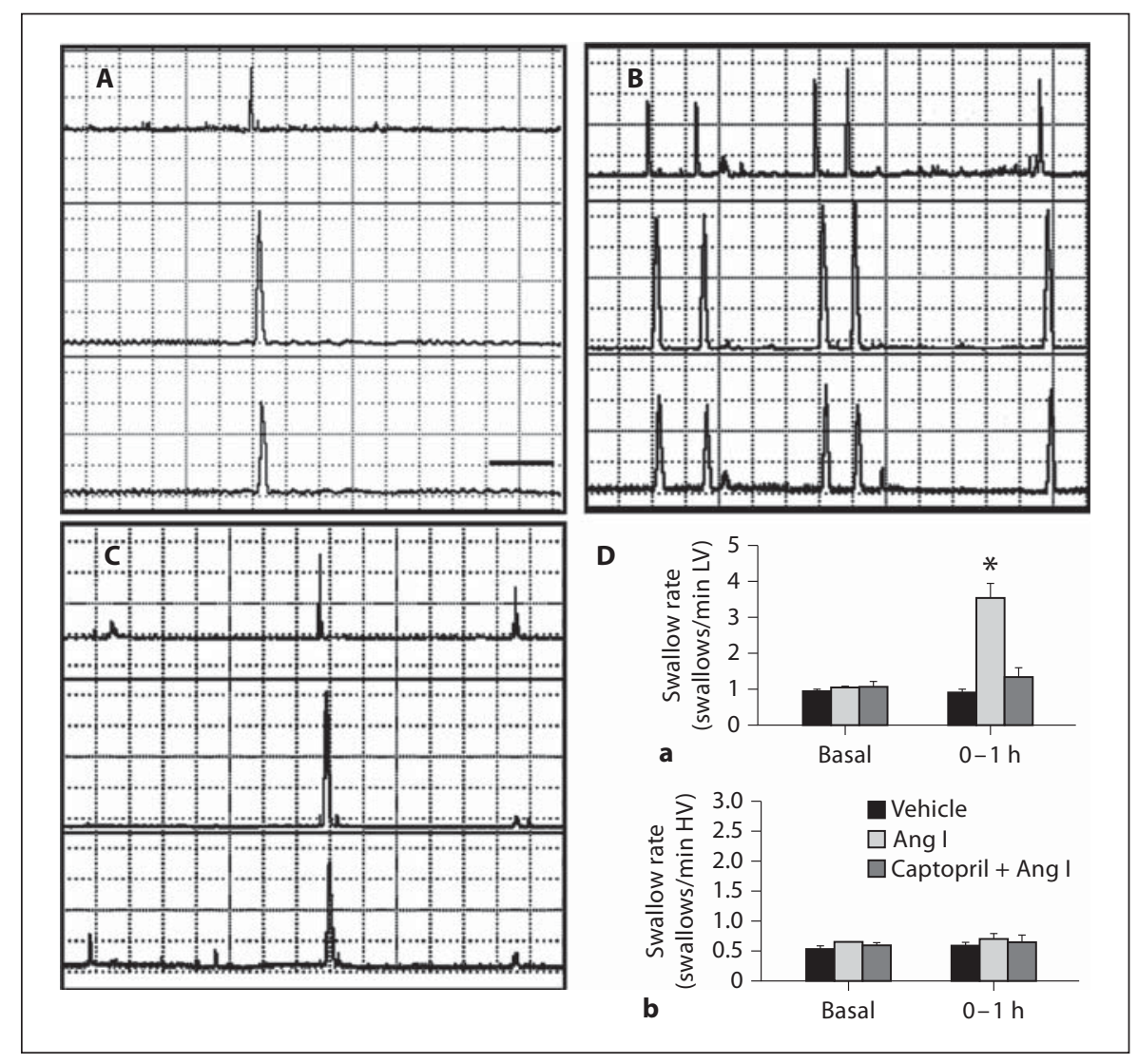

change from basal rates of $1.0 \pm 0.1$ swallows/min during LV ECoG and after i.c.v. vehicle injection (fig. 2A). However, after i.c.v. Ang I injection, the fetal swallowing during LV ECoG was increased to $3.5 \pm 0.4$ swallows/ min in the hour following administration $(\mathrm{p}<0.05$; fig. 2B). When captopril was administered centrally with Ang I, this did not change fetal swallowing during LV ECoG (fig. 2C). Figure 2D summarizes the fetal swallowing activity during LV (fig. 2Da) and HV (fig. 2Db) ECoG, before and after i.c.v. injection in three groups. As shown in figure $2 \mathrm{Db}$, the fetal swallowing activity during HV ECoG was not altered in either control or the experimental animals.

\section{Plasma OT Levels}

I.c.v. injection of vehicle, Ang I, or captopril with Ang I had no effect on plasma OT levels in maternal plasma (fig. 3), but fetal plasma OT concentrations were significantly $(\mathrm{p}<0.01)$ higher in the i.c.v. Ang I-injected fetuses than in control fetuses. I.c.v. Ang I increased $(p<0.01)$ fetal plasma OT levels. The peak level of plasma OT in fetuses was observed 15 min after i.c.v. injection of Ang
I, after which plasma OT levels declined. At 60 min after injection, the fetal plasma OT levels were still higher than the basal levels. In the animals treated with captopril and Ang I, there was a significant $(\mathrm{p}<0.01)$ inhibition of the Ang I-induced increase of fetal plasma OT concentrations. The fetal plasma OT levels in the captopril-treated group were similar to those of the control group.

\section{Fos-ir Staining}

In the control fetuses following injection with i.c.v. vehicle, there was no Fos-ir in the fetal forebrain structures, which included the SFO, MnPO, OVLT, SON, and PVN. However, i.c.v. injection of Ang I produced intense Fos-ir in these areas (fig. 4). There was a significant $(\mathrm{p}<0.01)$ difference in Fos-ir in the SFO, MnPO, OVLT, SON, and PVN between the i.c.v. vehicle- and the i.c.v. Ang I-injected fetuses. Ang I-induced Fos-ir in the PVN was detected in both magnocellular and parvocellular regions. In the captopril I-treated group, the number of Fos-ir cells in the SFO, MnPO, OVLT, SON, and PVN was the same as that in the controls. 


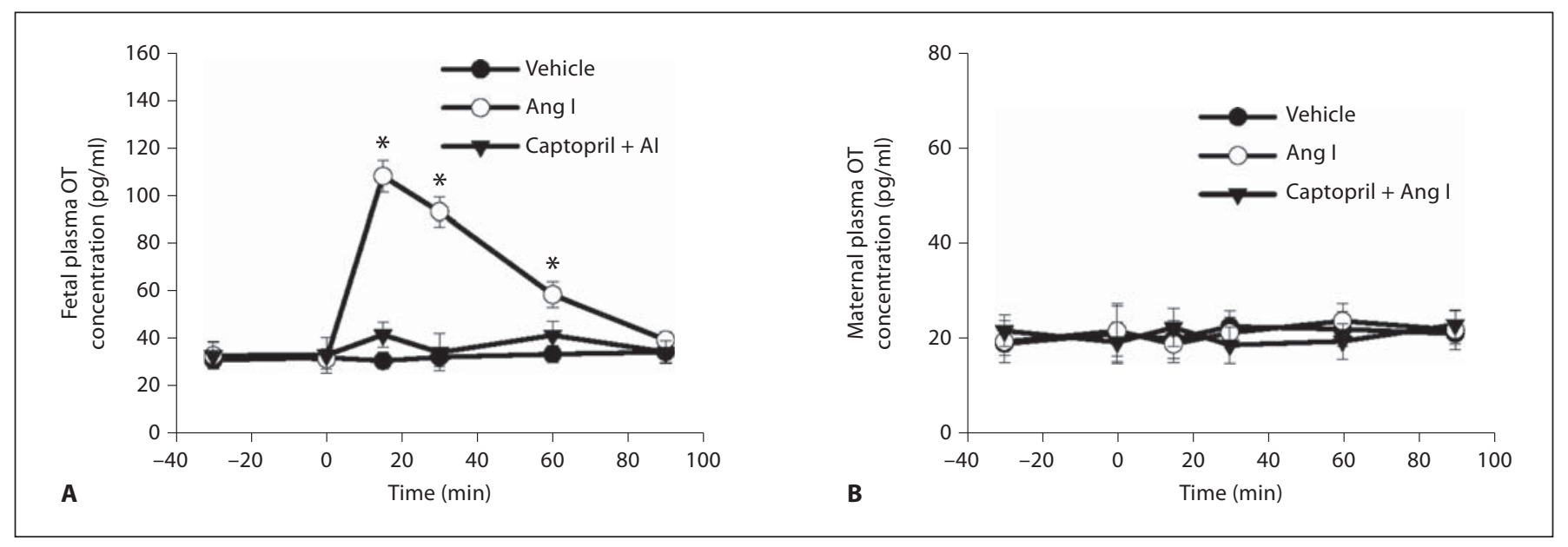

Fig. 3. The effect of fetal i.c.v. injection of vehicle, Ang I, or captopril with Ang I on fetal and maternal plasma OT concentrations. 0 min represents the time of i.c.v. injection. ${ }^{*} \mathrm{p}<0.01$ compared with the basal level.

\section{Fos-ir and OT-ir Double Labelling}

As shown in figure 5, OT-ir cells were located in the PVN and SON of the fetal hypothalamus. There was no Fos-ir in OT-containing cells in the brains of the control fetuses, but there was co-localization of Fos-ir in OT neurons in the PVN and SON of fetuses treated with Ang I (fig. 6). There was no difference in the total number of OT-ir cells in the PVN and SON of control and treated fetuses. Double labelling of Fos-ir and OT-ir in the PVN and SON was significantly $(\mathrm{p}<0.01)$ higher in the Ang I-treated fetuses than in the control animals, with approximately 38 and $35 \%$ of the OT neurons being labelled in the PVN and SON, respectively. In addition, double labelling of Fos-ir in the SON and PVN was significantly $(p<0.01)$ higher in the Ang I-treated fetuses than in the captopril-treated fetuses.

\section{Discussion}

This study demonstrates that i.c.v. Ang I produces Ang II-like dipsogenic and neuroendocrinological responses in the near-term ovine fetus in utero. I.c.v. injection of Ang I increased fetal swallowing activity occurring in LV ECoG without changing the temporal distribution of LV and HV ECoG. The neural activity labelled with Fos-ir in the putative salt appetite center, including the OVLT in the forebrain and the PVN in the hypothalamus, was enhanced. In addition, double labelling revealed that the central Ang I administration stimulated c-fos expression

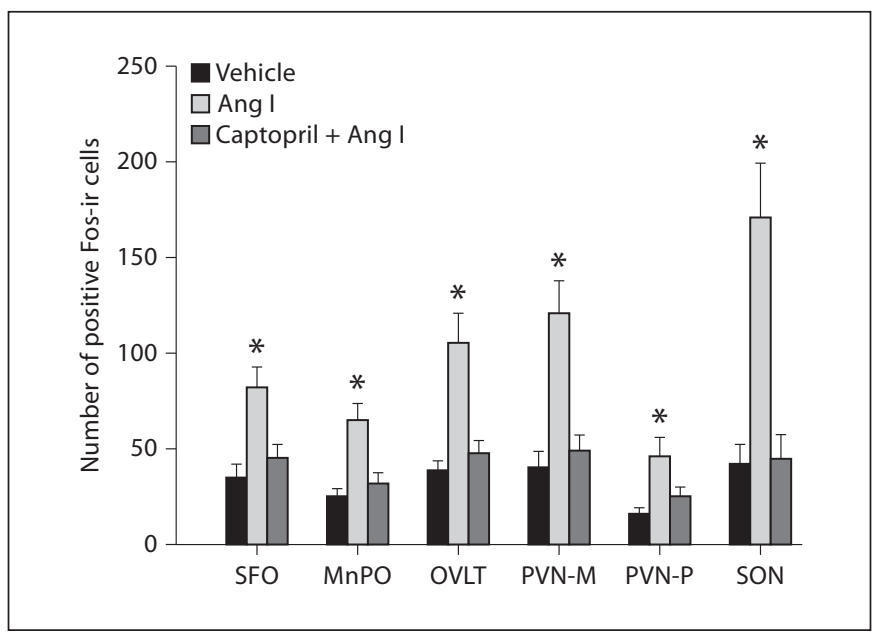

Fig. 4. Number of positive Fos-ir cells in the fetal brain after i.c.v. injection of vehicle, Ang I, or captopril with Ang I. ${ }^{*} \mathrm{p}<0.01 \mathrm{com}-$ pared to the control and captopril + Ang I i.c.v.-injected fetuses. $\mathrm{MnPO}=$ Median preoptic nucleus; OVLT $=$ organum vasculosum of the lamina terminalis; $\mathrm{PVN}-\mathrm{M}=$ magnocellular paraventricular nucleus; PVN-P = parvocellular paraventricular nucleus; $\mathrm{SFO}=$ subfornical organ; $\mathrm{SON}=$ supraoptic nucleus.

in the fetal OT neurons located in the PVN and SON in association with an increased fetal plasma OT. Furthermore, captopril, an inhibitor of ACE, significantly suppressed central Ang I-increased fetal swallowing and plasma OT. Together, the results demonstrate that ACE pathways in the fetal brain RAS are functional in the 

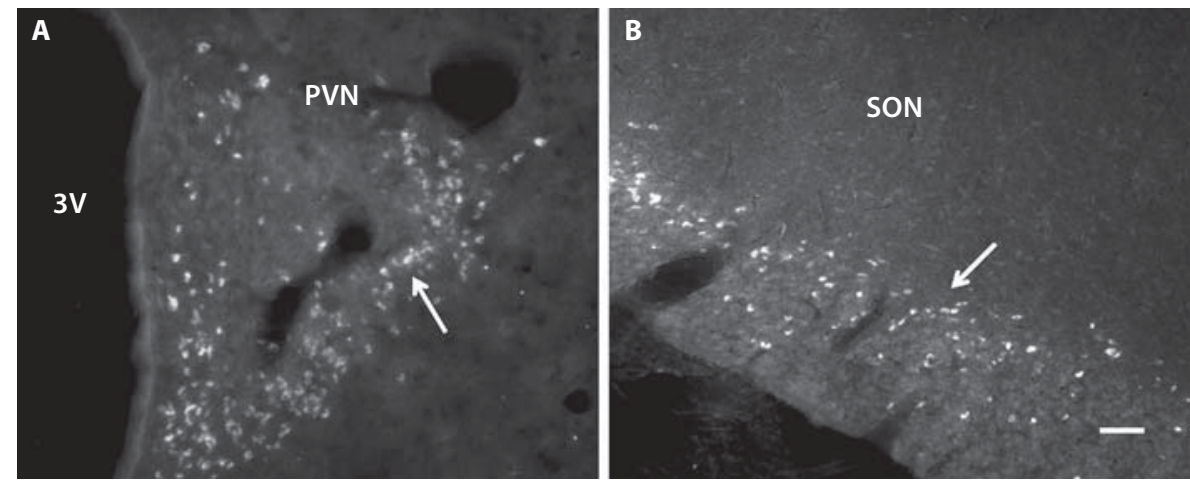

Fig. 5. OT-ir (+) cells in PVN and SON. Low-power fields of the PVN and SON. The white dots show the OT-ir (+) cells in the PVN (A) and SON (B). 3V = Third ventricle. Scale bar $=200 \mu \mathrm{m}$.
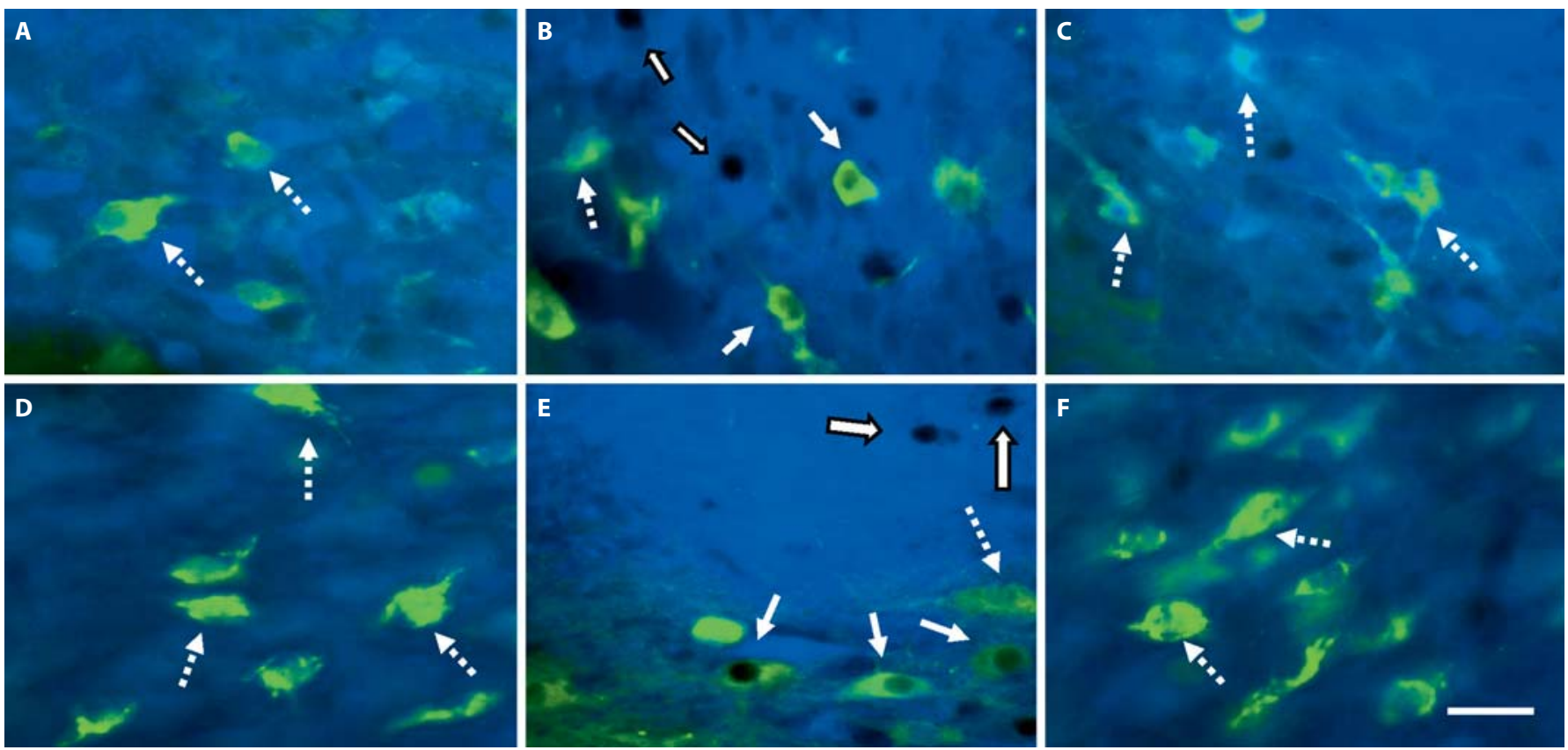

Fig. 6. Fos-ir and OT-ir in the PVN and SON following i.c.v. Ang I or captopril + Ang I injection. A-C: PVN. D-F: SON. A and D: i.c.v. vehicle. B and E: i.c.v. Ang I. C and F: i.c.v. captopril + Ang I. Solid white arrows indicate co-localization of Fos-ir and OT-ir.
Dashed white arrows indicate OT-ir positive cells without Fos-ir. Solid white arrows with black frame indicate positive Fos-ir outside of OT-ir-containing cells. Scale bar $=50 \mu \mathrm{m}$. third trimester of gestation, and exogenous Ang I may be converted into Ang II via endogenous ACE in the fetal brain to exert the dipsogenic and neuroendocrinological responses by acting at the sites consistent with the salt appetite neural network.

Ang II is a powerful signal for thirst and sodium appetite. When it is injected directly into the brain, Ang II causes an immediate water intake followed by a slower increase of $\mathrm{NaCl}$ intake [3]. Accumulating evidence shows that all components of RAS (including angiotensinogen, renin, ACE, AT 1 and AT 2 receptors) are expressed in the fetus in early gestation $[6,18,19]$. ACE is a key enzyme, and its mRNA and protein immunoreactivity have been detected in the fetal brain in both animals and humans [5-7]. However, whether and when the brain ACE in the fetus is functional during the fetal development remains largely unknown. The present study is the first to show the function of $\mathrm{ACE}$ in regulating body fluid homeostasis in ovine fetuses. 
Swallowing is an intrinsic fetal behavior, which develops in utero and contributes to fetal body fluid homeostasis. In ovine fetus, the swallowing response to dipsogenic signals is intact and functional at near-term $(0.85$ gestation) [8]. For instance, i.c.v. injection of Ang II enhanced fetal swallowing activity in near-term sheep fetuses [20]. This indicates that the fetal RAS has developed and is able to react to exogenous Ang II at near term. Fetal swallowing activity occurs in association with the ECoG activity, and alterations in fetal ECoG activity may affect fetal swallowing [21]. Because fetal swallowing occurs predominantly during LV ECoG periods [22], changes of the relative duration of $\mathrm{LV}$ periods may influence the swallowing rate.

In the present study, we observed that the percentage time of LV and HV ECoG did not change significantly following i.c.v. Ang I injection. The lack of change in fetal ECoG activity suggests a specific effect of central Ang I on the fetal swallowing behavior. Fetal swallows per minute of LV ECoG activity increased about 3.5-fold compared with the basal level after i.c.v. Ang I injection. This enhancement was similar to that evoked by i.c.v. exogenous Ang II as reported before $[15,20]$. Since Ang II is the main biological effector of RAS, and inactive Ang I must be converted to the active peptide to exert physiological effects, we hypothesized that the Ang II-like dipsogenic response following i.c.v. Ang I resulted from the production of Ang II in the local brain. To confirm this, pharmacological inhibition of the brain ACE was applied. A potent inhibitor of ACE, captopril [23], prevented the cleavage of the C-terminal His-Leu dipeptide from Ang I and blocked the formation of Ang II. The complete blockade of central Ang I-induced fetal swallowing activity by captopril strongly supports our hypothesis, indicating that $\mathrm{ACE}$ is functional in the body fluid regulation in the near-term fetal lamb.

Fetal swallowing may be regulated by multiple factors, including dipsogenic stimulation, appetite, hypertonicity, and behavioral status $[14,22,24,25]$. In the present study, the fetal physiological status remained stable as arterial values (particularly plasma osmolality and $\mathrm{Na}+$ concentrations) did not change following i.c.v. injection of Ang I, thereby excluding the possibility of contribution of systemic sodium/osmolality to the stimulated swallowing activity. The lack of change in arterial $\mathrm{PO}_{2}$ and $\mathrm{PCO}_{2}$ also excludes the possibility of hypoxia that might stimulate fetal swallowing as previously reported [26]. In addition to hypertonicity and hypoxia, hypovolemia and hypotension may also facilitate drinking behavior [27]. Our recent studies [28] showed that fetal mean arterial pressure was increased by i.c.v. Ang I. However, fetal swallowing after central application of Ang I was significantly increased without hypovolemia and hypotension. It is known that Ang II stimulates critical dipsogenic areas to elicit swallowing in the near-term fetuses. c-fos expression has been well described as a marker of the neuronal activation [29].

Mapping of c-fos allows the identification of individually active neurons in response to Ang I. The SFO and AV3V including MnPO and OVLT are demonstrated to be essential areas for thirst and sodium appetite, making extensive connections with the PVN in the hypothalamus [30-32]. In adults, these regions are richly distributed with AT $1 \mathrm{R}$ that responds to the dipsogenic action of Ang II. AT $1 \mathrm{R}$ has appeared in the major structures related to central cardiovascular and body fluid controlling pathways at the 0.7 of the gestational age [33]. In the present study, c-fos after i.c.v. Ang I was detected in the SFO, MnPO, and OVLT in the forebrain, and the PVN and SON in the hypothalamus. Captopril significantly inhibited Ang I-induced c-fos expression, suggesting that the neuronal activation was mediated by the pathway from Ang I to Ang II via ACE. The results reinforce our hypothesis that fetal brain endogenous ACE is functional, and it is able to convert exogenous Ang I into Ang II in dipsogenic regulation in the last third of gestation. In the present study, the patterns of c-fos expression after i.c.v. Ang I were generally consistent with those after i.c.v. Ang II application [15]. The activated regions included the SFO, MnPO, and OVLT in the forebrain, as well as the PVN and SON in the hypothalamus. Captopril significantly inhibited the c-fos expression induced by Ang I, suggesting that the neuronal activation was via Ang II (converted from Ang I by ACE) binding on AT 1 R. Associated with the stimulated swallowing activity by i.c.v. Ang I administration, the c-fos results reinforce our hypothesis that fetal brain endogenous ACE is functional and it can convert the exogenous Ang I to Ang II which acts on the critical dipsogenic areas to elicit dipsogenic behavior during the last third of gestation. In addition to the dipsogenic responses, central administration of Ang II may also induce the release of neurohypophysial hormones including VP and OT in adult animals in maintaining body fluid homeostasis [3]. As mentioned above, thirst responses after birth can be defined as water intake and salt intake in the regulation of body fluids. In acute thirst responses, Ang II causes a delayed and persistent increase in sodium appetite in adults. However, in fetal studies, it is technically difficult to distinguish salt intake from water intake in fetal swallowing. Despite this, both 
previous studies and our recent studies have shown that central Ang II increases VP release and the neural activity in the VP neurons in the fetal hypothalamus $[12,13]$, indicating that the central RAS-mediated neuropeptide network in the fetus is functional. Considering OT as a critical hormone involved in salt appetite and as a salt appetite-related biochemical signal in the brain, we focused on the hypothalamic OT system following i.c.v. Ang I stimulation in addition to detecting fetal behavioral responses.

It is now well established that OT plays an inhibitory role in regulating sodium appetite [3]. Central administration of OT decreases salt intake $[9,10]$. Studies in OT knock-out mice have shown that $\mathrm{OT}^{-/-}$mice display an enhanced salt appetite compared with $\mathrm{OT}^{+/+}$mice after water deprivation $[34,35]$, supporting the belief that OT has an inhibitory effect on sodium appetite. Moreover, central Ang II treatment not only stimulated OT release, but also activated central OT pathways that are inhibitory to salt appetite. Pretreatment of the animals with an OT receptor blocker increases the salt appetite in response to central administration of Ang II [11]. In the present study, although we cannot be certain that salt appetite changes in the fetus, the i.c.v. injection of Ang I caused an increase in fetal plasma OT concentrations. The fetal plasma OT increased by about 3.5 times the basal level and reached the maximum within $15 \mathrm{~min}$. The lack of change of the maternal plasma OT suggests that the increased fetal plasma OT is due to release from the fetal neurohypophysis in response to central Ang I and that the fetal OT does not pass the placental barrier to the maternal side. Although OT release may be induced by increases in osmolality and by hypovolemia [9], as mentioned above, the physiological status of the fetuses remained stable, which excludes such possibilities.
We propose that the increase in fetal plasma OT is due to the activation of OT neurons. OT is known to be synthesized and released from the magnocellular neurons defined as OT-producing cells, located in the PVN and SON (predominantly PVN) in the hypothalamus [3]. Our Fos immunostaining experiments revealed that there was intense Fos labelling in the cells of the fetal SON and PVN following i.c.v. Ang I. Double labelling showed the co-localization of Fos-ir and OT-ir in the fetal hypothalamus, demonstrating that the OT neurons were activated following Ang I injection.

Collectively, these data are consistent with our working hypothesis that endogenous brain ACE has functionally developed in the fetus in the third trimester of gestation in regulating body fluid homeostasis in utero. Given that drinking behavior and thirst responses play an important role in body fluid balance, and that they may be affected by adverse intrauterine environment during pregnancy, the novel information gained in the present study not only contributes to understanding of the normal neurophysiologic development in the fetal brain, but also provides insights in fetal programming of the RASmediated abnormalities of body fluid regulation.

\section{Acknowledgements}

This work was supported by NIH Grant (HL090920), Nature \& Science Foundation grants (31071033, 30973211, 8103006), Training program Foundation for Beijing Talents (2010D009010000001), Research Fund for the Doctoral Program of Higher Education of China (20101112120001), Scientific Research Foundation for the Returned Overseas Chinese Scholars by State Department of Human Resources and Social Security, and by Jiangsu Nature \& Science grants (BK2009122, 08KJB320013).

\section{References}

$\checkmark 1$ Ferguson AV, Wall KM: Central actions of angiotensin in cardiovascular control: multiple roles for a single peptide. Can J Physiol Pharmacol 1992;70:779-785.

2 Cunningham ET Jr, Sawchenko PE: Reflex control of magnocellular vasopressin and oxytocin secretion. Trends Neurosci 1991; 14:406-411.

3 Fitzsimons JT: Angiotensin, thirst, and sodium appetite. Physiol Rev 1998;78:583-686.

-4 Lang RE, Rascher W, Heil J, Unger T, Wiedemann G, Ganten D: Angiotensin stimulates oxytocin release. Life Sci 1981;29:1425-1428.

5 Tsutsumi K, Seltzer A, Saavedra JM: Angiotensin II receptor subtypes and angiotensin- converting enzyme in the fetal rat brain. Brain Res 1993;631:212-220.

-6 Schutz S, Le Moullec JM, Corvol P, Gasc JM: Early expression of all the components of the renin-angiotensin-system in human development. Am J Pathol 1996;149:20672079.

7 Strittmatter SM, Lynch DR, Synder SH: Differential ontogeny of rat brain peptidases: prenatal expression of enkephalin converytase and postnatal development of angiotensin-converting enzyme. Brain Res 1986;394: 207-215.

-8 Ross MG, Kullama LK, Ogundipe OA, Chan K, Ervin MG: Ovine fetal swallowing re- sponse to i.c.v. hypertonic saline. J Appl Physiol 1995;78:2267-2271.

-9 Stricker EM, Hosutt J, Verbalis JG: Neurohypophysial secretion in hypovolemic rats: inverse relation to sodium appetite. Am J Physiol Regul Integr Comp Physiol 1987; 252:R889-R896.

10 Stricker EM, Verbalis JG: Hormones and behavior. Biological basis of thirst and sodium appetite. Am Sci 1988;76:261-267.

- 11 Blackburn RE, Demko AD, Hoffman GE, Stricker EM, Verbalis JG: Central oxytocin inhibition of angiotensin-induced salt appetite in rats. Am J Physiol Regul Integr Comp Physiol 1992;263:R1347-R1353. 
12 Xu Z, Hu F, Shi L, Sun W, Wu J, Morrissey P, Yao J: Central angiotensin-mediated vasopressin release and activation of hypothalamic neurons in younger fetus at pre-term. Peptides 2005;26:307-314.

13 Xu Z, Shi L, Hu F, White R, Stewart L, Yao J: In utero development of central ANG stimulated pressor responses and hypothalamic cfos expression. Dev Brain Res 2003;145:169176.

14 Kullama LK, Agnew CL, Day L, Ervin MG, Ross MG: Ovine fetal swallowing and renal responses to oligohydramnios. Am J Physiol Regul Integr Comp Physiol 1994;266:R972R978.

15 Xu Z, Glenda C, Day L, Yao J, Ross MG: Central angiotensin induction of fetal brain c-fos expression and swallowing activity. Am J Physiol Regul Integr Comp Physiol 2001. 280:R1837-R1843.

16 Unger T, Kaufmann-Bühler I, Schölkens B, Ganter D: Brain converting enzyme inhibition: a possible mechanism for the antihypertensive action of captopril in spontaneously hypertensive rats. Eur J Pharmacol 1981;70:467-478.

17 Sherman DJ, Ross MG, Day L, Ervin MG: Fetal swallowing: correlation of electromyography and esophageal fluid flow. Am J Physiol Regul Integr Comp Physiol 1990; 258:R1386-R1394.

18 Butkus A, Albiston A, Alcorn D, Giles M, McCausland J, Moritz K, Zhuo J, Wintour EM: Ontogeny of angiotensin II receptors, types 1 and 2, in ovine mesonephros and metanephros. Kidney Int 1997;52:628-636.
19 Wintour EM, Alcorn D, Butkus A, Congiu M, Earnest L, Pompolo S, Potocnik SJ: Ontogeny of hormonal and excretory function of the meso- and metanephros in the ovine fetus. Kidney Int 1996;50:1624-1633.

20 Ross MG, Kullama LK, Ogundipe A, Chan K, Evin MG: Central angiotensin II stimulation of ovine fetal swallowing. J Appl Physiol 1994;76:1340-1345.

21 Nijland MJ, Chao CR, Ross MG: Anticholinergic suppression of ovine fetal swallowing activity. Am J Obstet Gynecol 1997;177: 1105-1112.

22 Harding R, Sigger JN, Poore ER, Johnson P: Ingestion in fetal sheep and its relation to sleep states and breathing movements. Q J Exp Physiol 1984;69:477-486.

23 Ferguson RK, Turini RK, Brunner GA, Garas $\mathrm{H}$, Mckinstry DN: A specific, orally active inhibitor of angiotensin-converting enzyme in man. Lancet 1977;1:775-778.

24 Brace RA, Wlodek ME, Cock ML, Harding R: Swallowing of lung liquid and amniotic fluid by the ovine fetus under normoxic and hypoxic conditions. Am J Obstet Gynecol 1994;171:764-770.

25 Xu Z, Nijland MJ, Ross M: Plasma osmolality dipsogenic thresholds and c-fos expression in the near-term ovine fetus. Pediatric Res 2001;49:678-685.

26 Sherman DJ, Ross MG, Day L, Humme J, Ervin MG: Fetal swallowing: response to graded hypoxemia. J Appl Physiol 1991;71:1856-1861.

27 Johnson AK, Edward GL: The neuroendocrinology of thirst: afferent signaling and mechanisms of central integration; in Pfaff DW, Ganten D (eds): Current Topics in Neuroendocrinology. Berlin, Springer-Verlag, 1990, pp 149-190.
28 Shi L, Mao C, Zeng F, Hou J, Zhang H, Xu Z: Central angiotensin I increases fetal AVP neuron activity and pressor responses. Am J Physiol Endocrinol Metab 2010;298:E1274E1282.

29 Hamamura M, Nunez D, Leng G, Emson P, Kiyama H: c-fos may code for a common transcription factor within the hypothalamic neuronal circuits involved in osmoregulation. Brain Res 1992;572:42-51.

30 Eng R, Miselis RR: Polydipsia and abolition of angiotensin induced drinking after 20 transections of subfornical organ efferent projections in the rat. Brain Res 1981;225: 200-206.

-31 Miselis RR, Shapiro RE, Hand PJ: Subfornical organ efferents to neural systems for control of body water. Science 1979;205:10221025.

- 32 Simpson JB, Routtenberg A: Subfornical organ: a dipsogenic site of action of angiotensin II. Science 1978;201:379-381.

$33 \mathrm{Hu}$ F, Morrissey P, Yao J, Xu Z: Development of $\mathrm{AT}(1)$ and $\mathrm{AT}(2)$ receptors in the ovine fetal brain. Dev Brain Res 2004;150:51-61.

34 Amico JA, Morris M, Vollmer RR: Mice deficient in oxytocin manifest increased saline consumption following overnight fluid deprivation. Am J Physiol Regul Integr Comp Physiol 2001;281:R368-R373.

35 Puryear R, Rigatto KV, Amico JA, Morris M: Enhanced salt intake in oxytocin deficient mice. Exp Neurol 2001;171:323-328. 